\title{
Expanding Imagined Affordance with Futuretypes: Challenging Algorithmic Power with Collective 2040 Imagination
}

\begin{abstract}
Imagined affordance speaks to the hidden affordances not often seen of user expectations. What if we asked a group of working class, ethnically diverse range of people to re-imagine alternative forms of digital platforms for 2040, and other time horizons? What would they re-imagine? How might this approach expand our set of theoretical constructs, methodologies in design practice for digital social media (DSM)? Student stories and our analysis of them comprise a hybrid of evidence interweaving design thinking with textual analysis and future studies. Informed by an analysis of social and technology trends, students explore the citizen imaginary for peerproducing alternative visions of our DSM. An analysis of student visions uncovers future memes of civil rights; platforms as new governing states; and resistance to algorithmic capture. These stories become a collective selfie into re-imagined social worlds. In their wake, futuretypes, signals of platform alternatives, emerge--an expanded range of citizen emotions, feelings, and desires.
\end{abstract}

\section{Introduction}

What if we asked a diverse group of working class and ethnically broad range of people to re-imagine alternative forms of digital social media and digital infrastructures for 2040? What would they offer or reimagine? How might this approach become part of a set of theoretical approaches and methodologies for envisioning design practice in digital social media (DSM)?

Lisa Nakamura [1] coined the term cybertype "to describe the distinctive ways that the Internet propagates, disseminates, and commodifies images of race and racism." I have coined the term futuretype, extending her work to encompass a broader range of issues of difference, promises of queer futurity and new political actors and subjects, with a focus on the ways in which ideological expectations, assumptions and biases are encoded into the stories we tell ourselves and one another about the future of digital social media. Lisa Nakamura and Peter Chow-White's edited book Race After The Internet [2] and Starship
Century, an edited anthology by James and Gregory Benford [3], serve as recent springboards for projecting ourselves into visions of new worlds. Based on these and similar texts, student stories reflect recent scientific discoveries and science fiction tropes as forecasting signals shaping our subjectivity, by reflecting our pasts and projecting what is to come.

As Peter Nagy and Gina Neff outline in discussing "imagined affordances" [4], the gap of imagination is the elephant in the room and often neglected in our research between how a citizen embraces and deploys a technology and what its designer intended. As Stuart Candy reminds us, we require a more sophisticated culture of the imagination [5], of intentional imagined insights not just from the designers, content strategists, and programmers at Facebook or Google. With more systematic attention to how personal stories of the future emerge, we can analyze the accompanying futuretypes they generate as the imagined affordances that elude and become discarded in most design processes.

We have the opportunity to tap into the best stories of imagined and re-imagined uses of our present network by directly creating a network of stories from the users and producers themselves, the produsers as Alex Bruns has labeled those who use social media and who contribute and mash up content for our digital culture daily [6]. In a number of experimental courses in organizational communication from 2008 to 2016 offered in one of the most diverse public state universities in the United States, students created detailed stories of DSM for imagined time horizons that spanned from 2025 to 2040 to 2112 to ten thousand years ahead to 12,012 C.E.!

This discussion offers accounts of what Gillespie [7] refers to as more interpretative flexibility for imagining nuances and new reconfigurations of algorithms and digital infrastructures. These stories convey artifacts embedded with a politics and set of values with the potential to stretch mindsets of those responsible for readying data for the algorithm. If each user and produser became a science fiction storyteller, they effectively become more adept in re-programming and perhaps hacking into the calculated publics they comprise. 
Too often we hear about the hidden and then publicized accounts of the industry prototype of new algorithmically driven applications. We interrogate the use of the term prototype into an expansive, aspirational term we call a futuretype within a grassroots frame for cultivating stories and uses of digital futures. The emerging movement of platform cooperativism argues for the digital platform as the basis and springboard for the renewal of the worker cooperative as the core of digital exchange rather than an extractive sharing economy. I propose that we create democratized forums for cooperatives of public imagination to convene regularly sponsored infusions of storied futures tinkering and repairing the civic damage wrought by an extractive monetization of our desires.

Alongside think tank and media visions of social media platforms, less visible are visions of ordinary citizens in discussing the future of social media platforms like Facebook. Certainly in user experience studies, Facebook and Google excel in gathering the minatiue of big data and narrow rationalistic studies of who clicks to whom for what reasons in the age-old paradigm of communication and user interaction studies. However, the real look and feel of future platforms and their algorithms reside in the social and imagined affordances that produsers are expecting, dreading, desiring, imagining and dreaming within a range of positive and negative valences. In a study of a sample of collected undergraduate, graduate student stories about their organizational, professional lives in 2040 and other future time projections as part of a multiyear project, this paper develops a theoretical and applied construct of the networked imagination to reflect the embodied, imagined affordances of next generation fears and dreams, the reservoir for reimagined algorithms where resistance, agency and hacking reside. The subjects in this study are from one of the most diverse public universities in the nation, predominately female, of color and from a mix of first generation college students, working class and middle class backgrounds [8].

A majority of these students work at retail jobs and most aspire to professional careers and struggle with the impatience to graduate, have their dream careers, and focus on the present and practical nature of balancing work and academic requirements. They are so overwhelmed by their present, they struggle with thinking about the future though it is continually on their minds as they voice concerns about social media influences on their private and public lives and the algorithms that will determine their job opportunities. Their stories act as springboards in creating organizational and digital network alternatives.
Few studies have asked the critical questions of those who feel they have less agency, of how they envision the future and navigate the ethical challenges of social media platforms, how their lives may coevolve with these platforms, and how their power might look for a plausible near term time horizon such as 2040 . Why not look at the dreams of real people and their collective imagination as an alternative and mirror alongside the calculated publics offered up by the platforms they use? By digging into the imagined affordances social media users employ, we have an opportunity to offer more diverse visions of how platforms can move beyond the hype of faux sharing to the next stage of humane and sustainable social capital and economies.

Our perspective methodologically aligns with, is inspired by Anne Balsalmo's call "taking culture seriously in the design and development of innovative technologies" [9]. We take the future stories crafted by our students seriously as an undervalued resource for technological innovation. Their technological imagination counts especially from a student body where ethnic minority voices predominate and that reside within an apartheid system of American education driven by property values, unequal resources and separated into public and private K-12 and university tracks, where minority voices are largely undervalued and under [10][11]. While many public universities dot the landscape of California, Silicon Valley companies routinely seek out and recruit nonminority students from private universities and confirm their own algorithmic hierarchies of largely white male power [11]. This stance is both dangerous and harmful for designing innovation for a nation demographically on track to become minority-majority by 2050 .

We work to unleash the imaginations of our students and to elevate the status of their dreams deferred where they have undervalued their own innovative qualities of mind "to transform what is known into what is possible" to think with technology to create impossible societies of equity, wealth and happiness [12]. We agree with Balsalmo [9] in cultivating imaginations resourceful in creating new democratic cultural organizations as much as we laud those who create new kinds of technologies and digital media. We hold that both occur simultaneously- that new imaginative cultural possibilities encourage new forms of media to facilitate their implementation. We too see ourselves as designers of experiential futures to conjure up a literacy of the technological imagination through enriched sensorial journeys into the future. Futuretypes capture daily routines, visual cues, haptic feel, and kinetic movement through an ethnography oriented to the future and the future of culture as 
bringing imagination into shape and embodied experience.

\section{Methodological approaches and data collection: creating the future world as a social imaginary and story to share}

Our methodological approaches evolved over this period (from 2008 to 2016) and began with creating imaginary social constructs, future imaginary worlds where students faced an organizational challenge to solve and explore as part of a final course project. We view the social imaginary, as a collective, shared vision of the future in the spirit of Benedikt Anderson and Cornelius Castoriadis [13][14]. We analyze student created stories as social imaginaries and as cultural artifacts to probe the interplay between emotion, digital media and its deployment [15][16][17]. Our platform for these constructs initially derived from a futures think tank, the Institute For The Future (IFTF) that offered forecasting games that the public and our students could access online for free. From that starting point and with the facilitation of our co-author, we created our own hypothetical imaginaries for students to use as scaffolds for their own creative thinking. A key insight involved the realization that students had to grapple with finding evidence to support their claims for their future imaginaries. We guided students in compiling evidence, outlining their stories, and providing license to re-imagine their lives. By late 2013, we converged on an optimal time horizon in our recent work as 2040 and made the outcome of the story more student-centered.

Indeed, students want to understand where they are personally going, what they might be doing and why and with whom. To that end, we asked students to envision their professional lives in 2040 in communication while simultaneously challenging them to design the organizational forms and digital media they might create, live within, and manage. From 2008 to 2016, we have collected hundreds of student stories to gain insight on their evolving snapshot into their collective and individual futures. We begin by tracing our methodological evolution, how students addressed a particular construct and what their imaginations reflected. We then outline the themes and emerging futuretypes that result from the samples of stories gathered, how their imaginaries expand or offer nuances into the standard visions of digital culture and their implications for digital social media design.

\subsection{Collecting stories and unleashing the right to imagine}

The Institute For The Future (IFTF) provided a model as additional precedence for scaling up collective visions. In 2008, IFTF launched the first massively multiplayer forecasting game known as Superstruct [18]. In lieu of our own interest to engage students' social imaginaries, we used the game as a platform for teaching about organizational change and transformation. Although the game is now an archived ghost town of gaming experiences, it foreshadowed ideas for storied collectivism and reframing anticipatory governance to re-think the opacity of algorithmic living. The word Superstruct itself is the opposite of 'destruct' and takes what is potentially stifling and unresponsive in organizational culture and explodes it into an alignment of multiple identities that superstruct, re-assemble, and heal the planet. The fantasy premise of the Superstruct game takes, as its starting point, the year 2019 where the fictitious Global Extinction Awareness System (GEAS) was set up to alert players to the likely time humans and life as we know it would cease. Based on what is dubbed the WorldRun Simulation, the GEAS predicted our extinction in 2042 unless we acted now. What it would take, the Superstruct narrative told us, was the reinvention of our organizations to enable us to collaborate from a small to a massive scale of extreme collaboration in order to face the five Superthreats: pandemics, technological power struggles, pervasive surveillance, massive relocations of people (global Diasporas), and environmental Armageddons (i.e., climate change, lack of drinking water, rising sea levels, and pollution). We refer to these themes as recurring, persistent futuretypes that shape and guide potential digital collaborative platforms in popular culture.

Players were asked to think up and create new startup communities, or what McGonigal [12] called superstructures, and to create new alliances of superstructures to handle these hypothetical superthreats. Points were gained by using a set of superhero powers, the same set of superhero powers cited earlier, on the path to becoming what McGonigal called a Super Empowered Human Individual or SEHI. As educators, we appreciated this term as translating empowered agency for our lower income, working student population.

Superstruct was introduced through a series of videos that oriented players to the future timeline of the game, and detailed each of the five superthreats, now prescient in their consistent reappearance in current news cycles. Players were invited to create their own media from blogs to videos about the imaginary superstructures they formed and the real alliances they made with other superstructures. Our students formed teams, considered a particular threat and imagined their 
superstructure. They had to consider the ideological and branding aims of their superstructure, as well to embed within it, a critical evaluation of power. A player's points increased with their collaborative activity. A wiki documenting Superstruct player activity went online to archive the inventive breadth of organizational brainstorming that ensued. Players who gained the most points had the opportunity to have their superstructures honored with an award in various categories from digital elite luminaries such as Wikipedia founder Jimmy Wales to science fiction writer Bruce Sterling.

Players invented superstructures with clever names and anthems. Our students' superstructures created a methodological precedent by prompting us to ask students to imagine larger social and collective structures in future design challenges. Out of the hundreds of superstructures created in the game, a few of our student visions provide an example of the satiric, organizationally subversive tone players and students created. Superstructures were meant to challenge the status quo; therefore, each title offered a reflective critique of the present. One of our student superstructures even gained commendation as a leading contender for Bruce Sterling's Veridian award for "the superstructure that creates the most irresistible demand for sustainable, or "green", design, entitled G.U.N. for Green United Nations. Our students played with our cultural penchant for violence by creating this acronym to take aim at ecological destruction in the search for more viable energy resources. The students explain:

GUNing for a sustainable future: Our system is marketed by having representatives from G.U.N approach nations around the world. Representatives first approach national leaders to discuss joining G.U.N to become a green energy source and distributor. Representatives form G.U.N also are looking at countries where new green energy sources can be found and then trying to locate scientists and technologists who can develop, distribute the new green sources of energy. These representatives, national leaders, scientists, technologists are joined together as a distributed intelligence group.

Another student team created R.E.B.E.L.: Our group is R.E.B.E.L (Radical Eminent Beyond Elevated Leaders); we want to get our message out to the general population and inform them about the surveillance and violation of their civil rights in our society in 2019. The government dominated our society. So we try our best to resist and defeat them. We created a magazine about the violations of our civil liberties that are occurring today--to win hearts and minds. We advertise our product the Stealth Chip...a chip that can be implanted in electronic devices that blocks outside electronic surveillance...Governments and Corporations cannot invade your personal life. (Student superstructure, 2008).

This post has a haunting and prescient quality about it by foreshadowing the influence of Wikileaks and
Edward Snowden's subsequent revelations about the National Security Agency pervasive surveillance programs. Its emphasis on marketing resistance presages ISIS's scaled up marketing of terrorism and Anonymous' manifesto for hacking. The superstructure ironically echoes conspiracy theories while highlighting their impending confirmation.

One student superstructure adopted an integrated approach to health care under the threat of the hypothetical pandemic disease REDS in the Superstruct narrative. Callings itself SHREDS, its tagline proclaims to educate "the public about REDS". A team member created its back story:

In 2019. I am a doctor working on the SHREDS team working to eradicate and educate people about REDS, a respiratory disease that is quickly becoming an epidemic...The internet, video games, and hologram virtual chat are the main culprits for causing the REDS epidemic. REDS thrives in the bodies of those who don't exercise and eat a healthy organic diet. These individuals don't have an immune system that can produce antibodies in response to REDS. These individuals end up taking concoctions of steroids and antibiotics that in turn make REDS take a new form and becomes resistant to medicine. I am working to educate the public on how to create a healthy life style through exercise and diet that will allow them to fight REDS without expensive medications and treatments. I am also working on revealing the importance of cannabis in the treatment of REDS and many other diseases. Cannabis is the miracle treatment for many diseases including REDS but the government won't allow it to be a widespread medication because it will devastate the drug industry and the billions of dollars that they collect from the drug companies will be lost...

This superstructure captures the zeitgeist of challenges to standard medical care and pharmaceutical pricing schemes that continue to resonate and inhabit countercultural and neoliberal rhetorical perspectives. The name SHREDS stands in for "shredding" weight as part of a fitness craze while simultaneously calling for "shredding" anti-marijuana laws as a veiled critique against government regulation. The student group buys into a countercultural, libertarian and neoliberal perspective. The continual refrain and research investigating how our digital devices might affect our health signal the skepticism and concern these students bring to this imaginary scenario. In creating their superstructures, students in turn at times reproduce themselves as subjects aligned with Silicon Valley perspectives of unregulated innovation. In this fashion, a countercultural, libertarian ethos perpetuates itself as an enduring futuretype to shred bureaucratic norms. 
Another prescient superstructure Hot Pink, speaks to 2016 immigration struggles:

Why pick Hot Pink? We don't treat people as objects that need to be relocated but as people with skills and talents that in turn can teach us and can help give back to the community locally, nationally and internationally. One of our services, a low rate credit card, is essential for new starters in the world of 2019 impacted by Generation Exile. (Student superstructure, 2008).

Hot Pink ironically sounds like a feminist call for radical inclusiveness using its name to play with the traditional, antiquated norms associated with certain gendered colors like pink. At the same time, the group stays within some rather conservative parameters by offering a credit card as their premium service; unlike the performance oriented guerilla antics of the Russian feminist group Pussy Riot, Hot Pink promotes telepresence and its own knowledge base dubbed HotPinkedia, their private digital network to galvanize their teams to aid refugee resettlement. They sought to guarantee welcoming stability.

A few superstructures even stuck with older organizational forms as a path to innovation. One nonstudent superstructure title that struck a humorous note: "Catholic Church-we've done this before." As researchers, our takeaway from the Superstruct experiment translated into allowing students to unleash and project their own particular biases, concerns, fears and desires as a legitimate aspect of the simulation and social imaginary to work through and address--to gain insight into the emotional spectrum of organizational and technical design.

Neff and Nagy recall that "for most users algorithms structure 'hidden affordances,' those of which users are unaware" [4] where "[a]ffordances 'include the expectations and beliefs of users, whether or not they are 'true' or 'right.' Affordances can and should be defined to include properties of technologies that are 'imagined' by users, by their fears, their expectations, and their uses, as well as by those of the designers. What people expect out of their data, the 'data valences' [19], are important aspects of the affordance of socio-technical systems"'[4]. Futuretypes, as persistent memes of the future, embody and circulate these imagined affordances of user aspiration.

Users or citizens are often unaware of how they might want to experience digital social media in the future and do not necessarily situate themselves as agents of sociotechnical change. One can argue that our fantasies for how digital networks and associated algorithms may evolve are part of an under-cultivated and "hidden affordance" of our collective imagination. Nevertheless, these collective dreams are present and inhabit the visible affordances that algorithms offer. Superstructures made these imagined affordances visible.
The themes from student superstructures and the hidden affordances they reveal comprise the futuretypes of emotions and desires we want to consider: G.U.N. and its violent acronym to take aim at averting ecological destruction; R.E.B.E.L. and its manifesto for protecting citizens against their own institutions as a quasi-government entity; SHREDS as an integrated health system of traditional science and holistic healing methods; Hot Pink as a superstructure for reframing immigrant status as a public good rather than as a threat. Their visions have held up over time, persist in various forms today and demonstrate a resilient working class ascendant in the breakout of rebellious sentiment for organizational, and by implication, digital social media redesign.

By collecting and analyzing student stories of their own future professional and organizational lives as they imagine them, we are tracing the mundane contours of the future normally not considered as important. Our work aligns with Sarah Pink's sensory ethnography [20] as we engage this population of students "as they live out the sensory, tacit, mundane and sometimes barely noticed elements of everyday life" [20] in sensory labs of the future. Superstruct as a game encouraged the use of homemade multimedia and featured IFTF made videos highlighting and conveying the superthreats that in turn stimulated players to convey their own sensorial journeys in staving off potential societal, human species collapse. The themes and narratives they create become the futuretypes "barely noticed" and the imagined affordances of emotional struggles glossed over in design practice. These mundane details constitute a challenge to algorithmic power by stretching the boundaries and interpretative flexibility for how algorithms can adjust to our sensual, emotional and irrational means of mapping the world. Futuretypes follow, reflect, grasp the double consciousness of future identities especially for those not in powerful elites. Student stories embrace W.E.B. Dubois' twoness [21] of operating among their own ethnic, gender, and class valences while grasping how to ascend or achieve status within a white, male dominated world.

\subsection{Building our own future imaginaries to engage students}

By 2012, we began creating our own future imaginary constructs for students to play with that involved organizational sustainability over ten thousand years for 12,012 C.E., and gender fluidity by 2112. In each case, students were asked to generate some form of collaborative superstructure embedded 
with imagined media and digital networked capabilities.

From 2012 to 2016, we systematically created a series of experimental courses with a focus on building a literacy in long term and futures thinking. These courses adopted an experiential futures approach based on our prior work in forecasting games where the players engage in an immersive challenge set in a future time. For one course in relational communication, our construct explored what would it take to create a sustainable organization able to remain viable long enough to protect ancient forests:

Design an Organizational Communication Strategy that Supports the Ancient Forest Consortia's Mission: restoring the world's ancient forests. Communication will need to be sustainable over the course of millennia. People will need to cooperate across generations.

A student team came up with an intriguing game: Legacy Tree. Their description seeks to maintain interest in forests through gaming:

One of our primary goals of Legacy Tree is to capture and maintain the interest of its participants so that the game may thrive throughout the course of thousands of years. Legacy Tree will introduce a series of threats to the trees, both hypothetical and real; players must continually participate in the game in order to maintain the well-being of their trees...they will acquire game credit, making them capable of avoiding and/or fighting any future threats...

Legacy Tree builds a competitive betting and prediction market for preservation. Through collaborative and self-interest, students succeeded in imagining a self-sustaining robust game to entice people to play over the long term. The pressure to consider the long-term sustainability of their collaborative venture ironically positioned the team to consider constant engagement as a tactic and as a quasi-religious set of rituals:

People can enter the ancient forest in their country, explore, plant a tree or choose an existing one to be named after and/or create a family tree plot.

This story construct for planning an organization capable of lasting to 12,012 C.E. was the most challenging time horizon for the project and engaged students in working out a set of rituals and gaming incantations to prolong their venture. They transformed the challenge into a spiritual quest and accented James Carey's insight into communication as ritual [22], perhaps the cornerstone to any robust futuretype is its ability to confirm and convey transcendence over time - an imagined affordance students described as a desirable quality amid rapid change.

Another course (in 2012) imagined Gender and Identity in 2112 where we offered students a design and entertainment challenge:

Gendered Lives 2112: In 2015 a group of neuroscientists, molecular scientists, and social researchers launch IDBIO, a design firm for Identity and Bio-Power...they explore, prototype the radical redesign of being human. Their innovation portfolio focuses on solutions for cognitive optimization, and gender design. They know that many of their products are edgy and may not be ready for prime time in Des Moines despite early success in early adopter markets in the San Francisco Bay Area, New York City, Tokyo. They are tasked to think 100 years in the future about the long-term implications of their bio-innovation innovation portfolio. Their core product areas include:

OptiCog Aids: a suite of digital tools and drugs that help people tune and train their brains to achieve optimum cognitive performance. A core principle of IDBIO's creative process is extreme provocation. To help them think about the very long term implications of their product groups they teamed up with Hollywood transmedia producers and create Gendered Lives 2112 a reality program in which a town in the U.S. adopts a suite of bioinnovations that dramatically change their human capacities and gender identification. Create a film treatment for an episode of Gendered Lives 2112:. Remember IDBIO wants to know what issues may emerge from widespread adoption of their products. Is this a time of a gender renaissance or dark age?

A student team looked at the amplification of transgender identity and how an OptiCog Aid gets rebranded as the GSwitch:

Al X stares at herself in the mirror, feeling fairly pleased with how G-Switch has worked. She feels like taking herself out to a club, but remembering some of the instructions that came with her GSwitch, she decides to start practicing her presentation. Initially she starts with how she stands and moves around laughing at herself as she adopts the more female-oriented physical behaviors of 2112. The club is a revelation, not least of which because it is specifically intended for people who [have] used G-Switch, euphemistically called "switches" (by people in favor of the use) and "flippers" (more derogatorily). On the way there, however, Al X gets called "flipgirl" by a pack of females who've chosen to express themselves by presenting stereotypically masculine and using products like ExoStrong. Al X, feeling a bit more vulnerable than she ordinarily might (both the change of gender, and being confronted by women presenting an extreme version of masculinity), mutters "boigrrls" under her breath as she hurries past (reflecting that language organizes perceptions of gender...). So by the time she gets to the club, she's feeling more nervous than usual. However, she's welcomed with open arms once she proves that she's used G-Switch.

The team was guided in their script by a transgendered student in teaching them how their own transition took place while imagining how digital 
media in 2112 could support their emerging identity as they experience -in and -out group hostility. Kim Stanley Robinson's work 2312 [23] similarly projects how human society becomes comfortable in its expansion in the solar system and in a radical expansion of genetically modified gender identities where G-Switch could have served as a precursor. None of the students knew of his work; instead their impetus to discuss transgender visions grew out of frustration with Facebook's former gender binary interface that failed to recognize more than two genders. By 2014, Facebook finally offered 58 options for gender identification [24].

2.3 Personalizing the social imaginary: the
professional life forecast and digital social
media
The next phase (from 2013 to 2016) in our methodology, as part of larger project in long term and futures thinking, focused on a final story assignment for a course in Organizational Transformation. Students developed an understanding of long term and futures thinking with regard to the rapidly changing work place and within organizational communication. By forecasting as peers together and crafting their own stories of their professional careers, students imagined the future of work in 2040 with an emphasis on commons-based peer production [25][6] where collaborative online work takes place on digital social networks and media across a variety of organizational contexts. We asked students to track significant news events in digital social media as a qualitative approach to indicate the probable forecasting significance for organizational structures, forms, and norms.

Students wrote responses to one of a number of provocative forecast questions: When will employers require cognitive augmentation of their employees? Or how will online social peer production or social network platforms evolve? By posing these questions at different times to different groups of students, the nature of our present digital infrastructure was indirectly handed over to them as an artifact to tinker with and re-imagine from its present configuration. These novice forecasters held little attachment for a specific Hollywood ending. Instead, their stories were similar to sandbox tools to craft new worlds and offer insight into their own concerns, worries, aspirations and to play them out with various dramatic or mundane endings.

Using the cone of uncertainty, a forecasting methodology defined by Paul Saffo [26], students explored what kinds of changes in work organization might emerge with what level of uncertainty. Our defining question for the cone is: How will online social networks evolve as part of our future workspace? Fall 2013 engaged students in forecasting an uncertain future and intentionally mapping known forms of digital collaboration in peer or what we call social production: collaborative ventures online that rely on networked micro-contributions from a Facebook "like" to collaborative social activism. This mapping consisted of highlighting likely trends, considering extreme logics if these trends continue, and exploring gray unknown gaps. Students charted their evolving ideas on large-sized butcher paper and as a course blog. Both platforms became our collective forecast.

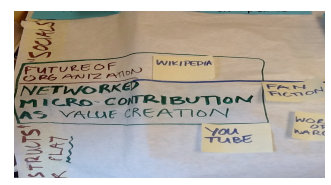

Figure 1: Cone of uncertainty map used in student forecasting of Commons-based peer production and digital social media.

We re-adapted Saffo's cone schematic by creating a cone of uncertainty mapping the future of social production (see Figure 1). The central trend defines what is already happening (indicators), what is likely to occur and least likely to happen (wild cards). Wild cards define the edges of the cone and forecasts. One student forecasted a more caring society based on social networking: "In the year 2040, the idea that an individual is responsible for...her own well being is considered cruel and barbaric. Instead work is given and done on a need basis." This forecast envisioned a supportive caring network for the United States: "Communal Care Organizations [are] becoming a new standard for providing most... of the necessities as well as most luxuries." Another proposed an open sourced legal network: "Law-for-All would allow lawyers to collectively work together for a common goal, providing social connections and social rewards." From creating a more caring society to an online collective legal network, students leveraged social production to address urgent civic dilemmas.

Based on their reading of social production trends and long term thinking, students brainstormed and clustered ideas about the reach of social production into our organizational lives shaping our relationships with authority, family and peers. Students posted large stickies of their ideas along the cone adding to the sensorial journey in making possible futures materialize (see Figure 2).

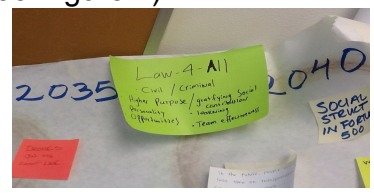

Figure 2: Student stickie forecast along the cone of uncertainty 
For their final project, students created a story that described a decisive moment in their professional life in 2040. They had to include a decisive moment into the story (i.e., such as being late to log into a virtual meeting) and how they negotiate this moment through the mediation of social networks. Their stories established a scene to open the story, the dialogue among people in the scene, the clues and context that conveys the year 2040, a decisive turning point and its resolution.

Dominant themes in student stories included: 1) collaborative social production through new media technologies imagining progressive social change (an open source legal system that pooled together legal expertise to free someone from the death penalty), 2) envisioning a caring society and system of cooperation, 3) critically forecasting an oppressive work system based on surveillance, 4) finding new niches for coaching face-to-face communication, within in an online context, and 5) the pros and cons of a reputation economy. These themes mark emerging and recurring futuretypes to reflect the lived and embodied hopes, fears, desires of an aspiring working class population of students struggling with digital social change while hailing their arrival into an altered and more elite status. Still, their dreams persist in working out their usually overlooked anxieties, annoyances, hopes and life chances in the networks they foresee. The story of Law-For-All imagined a new form of direct neurocommunication and mind connection embedded in an economy of social production: "At the peak of my excitement, I receive a BrainTime request from my brother. 'Accept request,' I exclaim. I express my excitement to my brother about Law-For-All's victory. His thoughts start pouring in. 'Congratulations! How does it feel? What's next?' I respond, 'Thank you. It feels...incredible. I learned so much. It's true what they say, it's amazing what can get accomplished when nobody cares who gets the credit'." For some, this exercise challenged their own sense of future work security and imagined solutions that envisioned stronger socio-economic safety nets. Forecasting confronted students' sense of "temporal exhaustion," Elise Boulding's term [28] [27] to denote a state where "if one is mentally out of breath all the time from dealing with the present, there is no energy left to imagine the future" We challenged students to move beyond their normal temporal frame. Students' imagined fictions act as records of their partial fantasies and intentional mapping of future networks.

The interpretative flexibility argued for by Gillespie [7] then depends on cultivating more imaginative possibilities to expand perceptual mindsets of algorithmic designers as well. "Imagined users are a 'mirror' to real users refracted through designers' "motivations and assumptions" and the necessity for augmenting that mirror with more visible citizen imagery of algorithmic foresight demands our attention. As Neff and Nagy insist, "[t]he point is not solely what people think technology can do or what designers say technology can do, but what people imagine a tool is for" [4]. Imagination entails perception, not just rationality.

The emphasis on affective states explains how student stories about imagined applications of digital social media may not reflect any technological mastery in understanding the mathematical formulas of algorithms. Instead, with some exposure to readings about social media, students create a mix of anchored materiality that reflect their values and emotional states. The storied futures of students reveal in more intimate detail the action of imagined affordances through the "emotional, affective, and "non-rational" elements of the relationship between users and technologies" [4].

\section{Collective Story as Collective Selfie: Student Citizen Imagined Affordances for 2040}

We wanted to bring our story methodology of 2040 to graduate students to see how their training and experiences might offer another perspective into storytelling digital social media. In graduate seminars in 2015 and 2016, students analyzed emerging signals of how social networks would look in 2040 and then created a story around those signals to convey an expanded range of emotional immersion into their 2040 world within two pages. In a story called Chin $U p$, one student imagined social media corporate platforms as new governing states superseding nation states where one's membership in Facebook becomes your primary citizenship. Taken to its logical extreme, nation states wither away as we tether ourselves to digital nations as our new governing platforms. Our allegiance to Apple, Chrome, Android and Facebook transitions us to a new style of citizenship as one student envisions:

In 2040, these social media states comprise the Alliance, a new union of social media platforms. Dual citizenship allows citizens of their original nation to join the ranks of digital nationhood as well. The Alliance is composed of the data superpowers with their grip over digital space recognized as territory [and] new order of social media states. (Student story, 2016).

Ironically, after the completion of this story, the New York Times and other media outlets ran a series of reports on Facebook's newfound power as a news curator with revelations about its small and undertrained Trending News staff making decisions as defacto editors although most had only training as 
engineers. Interrogating and expanding futuretypes and their imagined affordance can take further cues from the ubiquitous exchange of the torrent of selfies taken and managed daily. Selfies are apt metaphors for circulating stories and their accompanying imagery. Nancy Baym [29] for example asks "What precisely is a selfie?":

a photographic object that initiates the transmission of human feeling in the form of a relationship (between photographer and photographed...A selfie is also a practice-

The collective stories of students act similarly as a collective-selfie of projected images of anticipated living and social practices that have the potential to enter a digital collective space and become part of "a larger digital superpublic...created, displayed, distributed, tracked, and monetized through an assemblage of nonhuman agents" [29]. Similar to selfies, their stories embed politicized discourses about how people might represent, document, and share their anticipated, future behaviors. The story as collective selfie extends the mirror that designers and citizens could view, debate, and apply.

Another student imagined the lead up to a citizen rights network based on a fictitious racist and murder hate crime pushes the merger of Black Lives Matter and the hacker group Anonymous to join with each other in more coordinated resistance:

After her grandson dies at the hands of a racist bully...her tears...were interrupted by a...robotic sounding voice: We interrupt this broadcast, social media circuit, to bring you very important news.' The voice was eerily familiar. 'Ladies and Gentlemen of the United States, this is Anonymous...As stated by our friends of the Black Lives Matter movement, 'we are committed to...working vigorously for freedom and justice.'...we will continue to interrupt your lives; interfering with... social media outlets...

This vision points to a wider bridge between digital social protest networks and hacktivism to bend operating systems of current governance to their will.

The story goes beyond vigilante justice to ask for more good Samaritan watchfulness and the application of witnessing technologies to create:

a World Neighborhood Digital Network, conceived in the heart of a 33-year-old Programmer/Analyst who would climb out of her car on the freeway and ...shape the course of our lives... She was me. Fed up with watching our children die [...], I dreamed of a network that would unite us as national and global neighbors...

We point to working class and first generation college student imaginations as the hidden "minority reports" from the future that systematically comprise a collective resource for sustainable participatory voices in digital social media design and culture. Futuretypes carry resonant themes into daily practice as designs that embody multiethnic, gender, and class standpoints, voices and the nuances in-between.

\section{Conclusion: expanding imagined affordance and implications for digital social design [in progress]}

Envision workshops for Value Sensitive Design [30] offer insight into how the students' imagined futures can influence the actual design of future platforms. Student stories grouped by their persistent themes provide the means for creating a mobile digital platform of anticipated life and organizational pathways that others can reflect upon, edit and remix. By naming such an application as the Collective future selfie, this platform could as Value Sensitive Design cards, allow anyone by theme see a collective snapshot and perspective to generate and externalize visions of the future for various time horizons from 2025 to 12 , 016. How does surveillance look across these timelines? A corresponding database of futuretypes in science fiction can show matches between a student's vision and its resonance in popular culture. The higher the match could reflect the strong circulation of a particular futuretype and its emotional resilience. Before we begin repeating competitive based and other closed systems of collaboration, we want to make sure that student imagined futures uncover facets and nuances of the futuretype under consideration. I imagine the equivalence of an online card with a story that provokes fear for instance about pervasive surveillance and then flipping through a number of storied perspectives, adding one's own viewpoint with the goal of seeing that fear quotient transform into a desire to generate a new service, new app, or cooperative-superstructure.

We see the compilation of imagined stories as a guide to design and draw inspiration from Jane McGonigal's call for "our collective work...to make what seems impossible" such as equity of access to health and education possible. "To make the utopian practical"'[12]. As McGonigal notes, the storyteller Maurice Sendak once said, "In order to work, fantasy must be rooted 10-feet deep in reality" and these stories offer the reality check of demanding user and produser expectations, desires, and emotions to fulfill our dreams.

\section{References}

[1] Nakamura, L. (2013). Cybertypes: Race, ethnicity, and identity on the Internet. New York, NY: Routledge. 
[2] Nakamura, L., \& Chow-White, P. (Eds.). (2013). Race after the Internet. New York, NY: Routledge.

[3] Benford, G. (2013). Starship Century: Toward the Grandest Horizon. :Lucky Bat Books.

[4] Nagy, P., \& Neff, G. (2015), "Imagined Affordance: Reconstructing a Keyword for Communication Theory", Social Media+ Society, 1(2), Sage, New York, 2015, 2056305115603385.

[7] Gillespie, T. (2014), 9 "The Relevance of Algorithms",Media technologies: Essays on communication, materiality, and society, MIT Press, MA, 2014, pp. 167.

[8] 2014 Almanac of Higher Education: Diversity (2014). Chronicle of Higher Education.

[9] Balsamo, A. (2011). Designing culture: The technological imagination at work. Durham, NC: Duke University Press.

[10] Giroux, H. A., \& Giroux, S. S. (2004). Take back higher education: Race, youth, and the crisis of democracy in the post-civil rights era. Macmillan.

[11] Bui, Q and Miller, C.C. (2016, February, 25). Why Tech Degrees Are Not Putting More Blacks and Hispanics Into Tech Jobs. The New York Times. Retrieved from http://www.nytimes.com/2016/02/26/upshot/dont-blamerecruiting-pipeline-for-lack-of-diversity-in-tech.html?_r=0

[12] Institute For The Future. (2013, April 26,). Framework: Public Imagination. Reconstitutional Convention Palo Alto, CA. Retrieved from

http://reconcon.govfutures.org/?recent_works=framewo rks8

[13] Anderson, B. (2006). Imagined communities: Reflections on the origin and spread of nationalism. Verso Books.

[14] Elliott, A. (2002). The social imaginary: A critical assessment of Castoriadis's psychoanalytic social theory. American Imago, 59(2), 141-170.

[15] Marcus, G. E. (1998). Corporate futures: The diffusion of the culturally sensitive corporate form (Vol. 5). University of Chicago Press.

[16]Antonopoulou, A., \& Dare, E. (2013). The digital dreamhacker: crowdsourcing the dream imaginary. The Sydney eScholarship Repository. Proceedings of the 19th International Symposium on Electronic Art, ISEA2013, Sydney. Retrieved from: http://hdl.handle.net/2123/9749

[17] Boehner, K., DePaula, R., Dourish, P., \& Sengers, P. (2007). How emotion is made and measured. International Journal of Human-Computer Studies,65(4), 275-291.
[5] Candy, S., The Futures of Everyday Life: Politics and the Design of Experiential Scenarios (Doctoral dissertation). Department of Political Science, University of Hawaii at Manoa, 2010.

[6] Bruns, A. (2008). Blogs, Wikipedia, Second Life, and Beyond: From Production to Produsage. New York, NY: Peter Lang

[18] Institute For The Future (2008). Explore the World of Superstruct. Retrieved from http://www.iftf.org/our work/people-technology/games/superstruct/

[19] Fiore-Gartland, B., \& Neff, G. (2015). Communication, mediation, and the expectations of data: data valences across health and wellness communities.International Journal of Communication, 9, 19.

[20] Pink, S. (2015). Doing sensory ethnography. London, UK: Sage.

[21] Du Bois, W. E. B., \& Edwards, B. H. (2008). The souls of black folk. Oxford, UK: Oxford University Press.

[22] Carey, J. W. (2008). Communication as culture, revised edition: Essays on media and society. New York, NY: Routledge.

[23] Robinson, K. S. (2012). 2312. London, UK: Hachette UK.

[24] Goldman, R. (2014). Here'sa list of 58 gender options for Facebook users. ABC News Blogs, 13.

[25] Benkler, Y., \& Nissenbaum, H. (2006). Commons-based peer production and virtue. Journal of Political Philosophy, 14(4), 394-419.

[26] Saffo, P. (2007). Six rules for effective forecasting. Harvard business review,85(7/8), 122.

[27] Brand, S. (2008). Clock of the long now: Time and responsibility. Basic Books.

[28] Boulding, E. (1978). The dynamics of imaging futures. World Future Society Bulletin, 12(5), 1-8.

[29] Senft, T. M., \& Baym, N. K. (2015). What does the selfie say? Investigating a global phenomenon introduction.

[30] Friedman, B. (1996). Value-sensitive design. interactions, 3(6), 16-23. 